\title{
The celiac seagull
}

\author{
Siu Chun Wong $\odot,{ }^{1}$ Yan-Lin $\mathrm{Li}^{2}$ \\ ${ }^{1}$ Department of Radiology, Princess Margaret Hospital, Kowloon, Hong Kong \\ ${ }^{2}$ Department of Radiology, Queen Mary Hospital, University of Hong Kong, Pokfulam, Hong Kong
}

The celiac "seagull" (Fig. 1) refers to the division of the celiac trunk into the hepatic artery and splenic artery, which resembles a seagull soaring (Fig. 2).

Anatomically, the celiac artery arises from the abdominal aorta from level of the twelfth thoracic vertebra and trifurcates into the common hepatic, splenic artery, and the left gastric artery.

The "seagull" is observed in a transverse plane over the epigastrium, and serves as a useful landmark for identification of the celiac trunk [1].

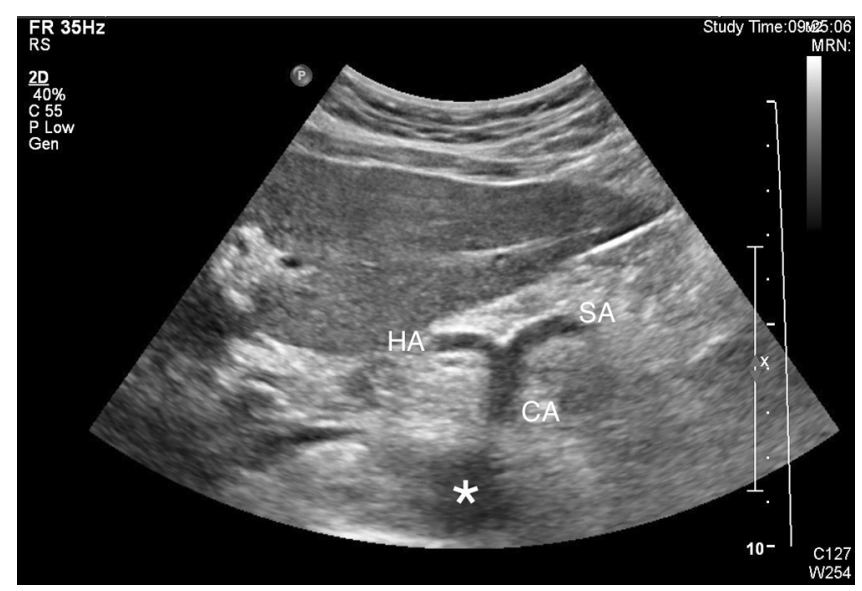

Fig. 1. Transverse abdominal ultrasound image demonstrating the bifurcation of the celiac artery (CA) into the hepatic artery (HA) on the right and splenic artery (SA) on the left, resembling a seagull. The asterisk $\left({ }^{\star}\right)$ denotes the abdominal aorta.

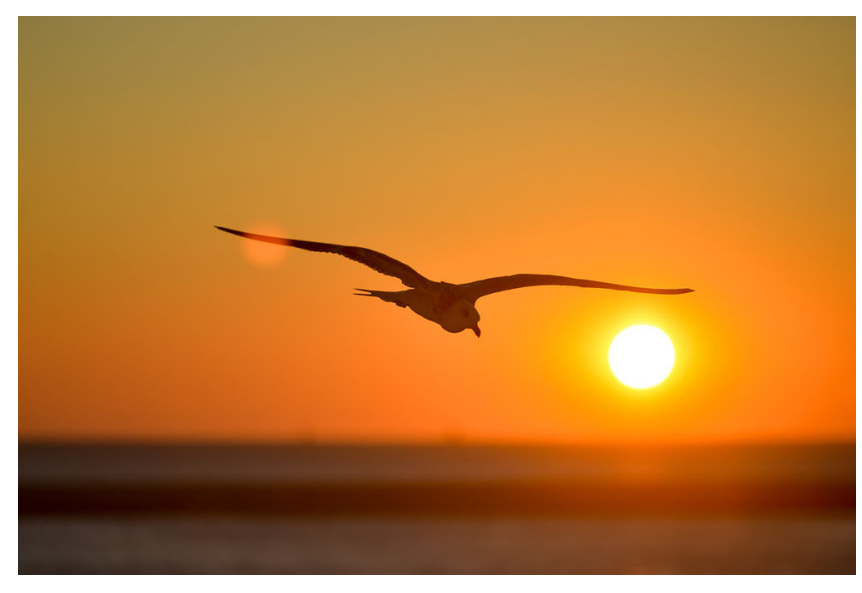

Fig. 2. A seagull stretching its wings [2].

Compliance with ethical standards

Funding No funding was received for this study.

Conflict of interest The authors declare that they have no conflict of interest.

Ethical approval This article does not contain any studies with human participants or animals performed by any of the authors.

Informed consent Statement of informed consent was not applicable since the manuscript does not contain any patient data.

\section{References}

1. Alty J, Hoey E, Wolstenhulme S, et al. (2013) Practical ultrasound: an illustrated guide, 2nd edn. CRC Press, Boca Raton.

2. Photo Courtesy of Free-Photos. https://pixabay.com/en/seagullanimal-bird-backlight-601287/. Accessed 30 Jan 2018. 